\title{
A Hubble Space Telescope Survey for Novae in M87. II. Snuffing out the Maximum Magnitude-Rate of Decline Relation for Novae as a Non-standard Candle, and a Prediction of the Existence of Ultrafast Novae*
}

\author{
Michael M. Shara ${ }^{1,2}$, Trisha Doyle ${ }^{1,3}$, Tod R. Lauer ${ }^{4}$, David Zurek ${ }^{1}$, Edward A. Baltz ${ }^{5}$, Attay Kovetz ${ }^{6}$, Juan P. Madrid ${ }^{7}$, \\ Joanna Mikołajewska ${ }^{8}$, J. D. Neill ${ }^{9}$, Dina Prialnik ${ }^{10}$, D. L. Welch ${ }^{11}$, and Ofer Yaron ${ }^{12}$ \\ ${ }^{1}$ Department of Astrophysics, American Museum of Natural History, Central Park West and 79th Street, New York, NY 10024-5192, USA \\ 2 Institute of Astronomy, University of Cambridge, Madingley Road, Cambridge CB3 OHA, UK \\ ${ }^{3}$ Department of Physics and Space Sciences, Florida Institute of Technology, Melbourne, FL 32901, USA \\ ${ }^{4}$ National Optical Astronomy Observatory, P.O. Box 26732, Tucson, AZ 85726, USA \\ ${ }^{5}$ KIPAC, SLAC, 2575 Sand Hill Road, M/S 29, Menlo Park, CA 94025, USA \\ ${ }^{6}$ School of Physics and Astronomy, Faculty of Exact Sciences, Tel Aviv University, Tel Aviv, Israel \\ CSIRO, Astronomy and Space Science, P.O. Box 76, Epping, NSW 1710, Australia \\ ${ }_{9}^{8}$ N. Copernicus Astronomical Center, Polish Academy of Sciences, Bartycka 18, PL 00-716 Warsaw, Poland \\ ${ }^{9}$ California Institute of Technology, 1200 East California Boulevard, MC 278-17, Pasadena CA 91125, USA \\ ${ }^{10}$ Department of Geosciences, Tel Aviv University, Ramat Aviv, Tel Aviv 69978, Israel \\ ${ }^{11}$ Department of Physics \& Astronomy, McMaster University, Hamilton, L8S 4M1, Ontario, Canada \\ 12 Department of Particle Physics and Astrophysics, Weizmann Institute of Science, 76100 Rehovot, Israel \\ Received 2016 December 21; revised 2017 February 19; accepted 2017 March 7; published 2017 April 21
}

\begin{abstract}
The extensive grid of numerical simulations of nova eruptions from the work of Yaron et al. first predicted that some classical novae might significantly deviate from the Maximum Magnitude-Rate of Decline (MMRD) relation, which purports to characterize novae as standard candles. Kasliwal et al. have announced the observational detection of a new class of faint, fast classical novae in the Andromeda galaxy. These objects deviate strongly from the MMRD relationship, as predicted by Yaron et al. Recently, Shara et al. reported the first detections of faint, fast novae in M87. These previously overlooked objects are as common in the giant elliptical galaxy M87 as they are in the giant spiral M31; they comprise about $40 \%$ of all classical nova eruptions and greatly increase the observational scatter in the MMRD relation. We use the extensive grid of the nova simulations of Yaron et al. to identify the underlying causes of the existence of faint, fast novae. These are systems that have accreted, and can thus eject, only very low-mass envelopes, of the order of $10^{-7}-10^{-8} M_{\odot}$, on massive white dwarfs. Such binaries include, but are not limited to, the recurrent novae. These same models predict the existence of ultrafast novae that display decline times, $t_{2}$, to be as short as five hours. We outline a strategy for their future detection.
\end{abstract}

Key words: binaries: close - novae, cataclysmic variables

\section{Introduction and Motivation}

Most astronomers associate Edwin Hubble with the year 1929 because of his momentous paper, "A Relation between Distance and Radial Velocity among Extra-Galactic Nebulae" (Hubble 1929a), which initiated the study of modern cosmology. In the same month, however, Hubble also published "A Spiral Nebula as a Stellar System, Messier 31" (Hubble 1929b), in which he announced the resolution of the outer spiral arms of that galaxy into swarms of faint stars, the discovery of Cepheids and long-period variables, and 63 novae. The study of extragalactic stellar populations thus began at the same time as cosmology, and classical novae have played a significant role in the study of populations ever since.

Novae are all binaries in which a white dwarf (WD) accretes matter from a hydrogen-rich brown dwarf, red dwarf or red giant companion, or helium from a WD companion. When sufficient mass is accumulated that degenerate electron pressure at the base of the accreted envelope exceeds a critical value, a thermonuclear runaway (TNR) occurs that ejects most of the envelope and brightens the WD to its Eddington luminosity or

\footnotetext{
* Based on observations with the NASA/ESA Hubble Space Telescope, obtained at the Space Telescope Science Institute, which is operated by AURA, Inc., under the NASA contract NAS 5-26555.
}

even brighter (Shara 1981). Novae near maximum light range in luminosity from $M=-6$ to $M=-10$ (Warner 1995). In contrast, the bright end of the planetary nebula (PN) luminosity function only reaches $M(5007)=-4.5$ (Ciardullo et al. 1989), red giant branch (RG) stars typically reach $M=-3$ (Baade 1944; Sandage 1971), and RR Lyrae stars achieve $M_{V}=0.6$ (Christy 1966). Novae can therefore be detected more easily in a given galaxy or cluster, and can be observed to significantly greater distances in the field than PN, RG, or RR Lyrae stars. The transient nature of novae and their $\mathrm{H} \alpha$ brightness help eliminate contamination due to background emission line objects or unresolved compact galaxies.

Zwicky (1936) was the first to announce that novae appeared to behave as standard candles, with light curves that could be calibrated to yield the distances to galaxies. Zwicky's first formulations of the Maximum Magnitude-Rate of Decline (MMRD) correlation were improved upon by Mclaughlin (1945) and Arp (1956). The physics of the apparently tight correlation between nova absolute magnitude at maximum light and their rates of brightness decline was explained by Shara (1981) and Livio (1992). The key prediction of those investigations is that, all other things being equal, the mass of the WD in a nova binary is the dominant parameter controlling the behaviors of novae explosions. 
The essential physics underlying this prediction is as follows. The degenerate equation of state of matter in a WD determines that as the mass of a WD increases, its radius decreases (Chandrasekhar 1931, 1935). Thus the acceleration of gravity at a WD's surface increases sharply as its mass increases. A strongly increasing gravitational potential, with increasing WD mass, means that much less hydrogen can be accreted onto the WD before a TNR occurs (Shara 1981). Envelopes with lower mass can be ejected faster than those of higher mass, so novae occurring on massive WDs will exhaust their thermonuclear-powered envelopes, and decline in brightness faster than those on low-mass WDs. If WD mass was the only free parameter, then novae would be luminous, wellunderstood standard candles displaying negligible scatter.

Of course, all other things are not equal, and novae are decidedly not a phenomenon governed by just one free parameter (Starrfield et al. 1975; Shara et al. 1980). While it is widely recognized that WD mass is a critical factor affecting nova explosions, it is now understood that there are at least four other important factors that determine the properties of a nova outburst. These factors are the accretion rate onto the WD and the resulting envelope mass (Prialnik et al. 1982), the WD luminosity (Prialnik \& Kovetz 1995; Yaron et al. 2005), its chemical composition ( $\mathrm{He}, \mathrm{CO}$, or $\mathrm{ONe}$ ), and the chemical composition of the accreted matter (H-rich or He; Faulkner et al. 1972; Kovetz \& Prialnik 1985; Starrfield et al. 1986). Just the WD mass, accretion rate/envelope mass, and luminosity can, and do, produce a rich variety of nova eruptions and scatter about the so-called MMRD relation (Prialnik \& Kovetz 1995; Yaron et al. 2005; Hachisu \& Kato 2010). Referring to their nova models, Prialnik \& Kovetz (1995) stated that "Correlations are obtained between the peak luminosity and time of decline...It is shown that these correlations cannot be tight...The implication is that novae cannot be considered accurate distance indicators." Do observations bear out this prediction?

Early attempts to measure distances of nearby galaxies, and even to deduce the Hubble constant via novae, have had a reasonably good track record. Capaccioli et al. (1989) used Cohen's (1985) calibration of Galactic novae to obtain a distance modulus for M31 of $24.27 \pm 0.2$, in good agreement with $(m-M)=24.46 \pm 0.10$, recently obtained by de Grijs $\&$ Bono (2014). Capaccioli et al. (1990) found a distance to the LMC of $(m-M)=18.7 \pm 0.2$, in equally good agreement with the modern value of $18.48 \pm 0.10$ (Inno et al. 2016). della Valle \& Livio (1995) used the M31 and LMC MMRDs to obtain a Virgo cluster distance of $18.6 \pm 3.3 \mathrm{Mpc}$, which exceeds the modern distance of $16.4 \pm 0.5 \mathrm{Mpc}$ by $13 \%$ (Bird et al. 2010). Finally, using a sample of just seven novae, a value of the Hubble constant of $70 \pm 13 \mathrm{~km} \mathrm{~s}^{-1} \mathrm{Mpc}^{-1}$ was obtained by van den Bergh (1992). della Valle \& Livio (1995) summarized these studies, stating that novae can be used judiciously, when geometric and nebular parallaxes are not available, resulting in roughly $30 \%$ errors in distance measurements to non-recurrent Galactic novae. In the modern era of precision cosmology, novae are not competitive with much more precise values of the Hubble constant obtained via SNe Ia (e.g., Riess et al. 2011), but 25 years ago the MMRD seemed to be a much better distance indicator than the pessimistic assessment of Prialnik \& Kovetz (1995) then indicated.
Doubts about the MMRD relation were first raised by Ferrarese et al. (2003, p. 1302), who noted that,

"We examine the maximum magnitude versus rate of decline (MMRD) relation for novae in M49, finding only marginal agreement with the Galactic and M31 MMRD relations. Up to six of the nine novae detected with the Hubble Space Telescope (HST) in this study appear to be anomalously faint for their fast rates of decline, but conclusive maxima were only seen for three of the nine novae."

A similar conclusion was reached the following year by Hearnshaw et al. (2004, p. 106), who, on the basis of 4 wellobserved fast novae in the LMC concluded,

"The weighted mean distance modulus to the LMC based on these novae is $18.89 \pm 0.16$. This differs significantly from the distance modulus adopted by della Valle \& Livio of $18.50 \ldots$ The evidence based on these novae suggests that... some novae in the LMC, including these four, are significantly underluminous at maximum light compared with those in M31, by about 0.4 mag."

The strongest recent objection to MMRD came when Kasliwal et al. (2011) achieved a major breakthrough with their monitoring of M31 for novae and the resulting observational discovery of "faint, fast novae." Their nightly cadence (except when interrupted by weather) and relatively deep magnitude limit overcame the observational bias against the discovery of such faint, fast transients, that was inherent in all previous nova surveys. Rather than being rare outliers, these novae were a significant fraction of all M31 novae detected. Referring to these novae, Starrfield et al. (2016) stated that "...a single-valued MMRD relationship does not exist as is shown by observations (Kasliwal et al. 2011)." In the past year (Shara et al. 2016) have shown that these faint, fast novae are as common in M87 as they are in M31.

In Section 2 we summarize observations of well-observed novae in the Milky Way, LMC, M31, M33, and M87. We plot the maximum luminosities of novae in these five galaxies versus $t_{2}$, the time to decline $2 \mathrm{mag}$, in Section 3, showing that the MMRD should be discarded as a distance indicator. In Section 4 we use the extensive grid of nova models in Yaron et al. (2005) to explain the observed large observational scatter in the MMRD, and determine which nova parameters give rise to faint, fast novae. We predict the existence of ultrafast novae with $t_{2}<1$ day in Section 5. Our conclusions are summarized in Section 6.

\section{Observations}

A compilation of the peak magnitudes and distances to, and hence peak luminosities of 28 Galactic novae is given by Downes \& Duerbeck (2000). These authors used ground-based and HST images of shells and a mix of both their own and literature spectroscopic expansion velocities to determine expansion parallax distances to the largest uniformly analyzed sample of Milky Way novae in the literature. We adopt their absolute magnitudes and $t_{2}$ times to decline from maximum brightness for Milky Way novae, and add the Galactic symbiotic nova $\mathrm{T} \mathrm{CrB}$ because of its equally well determined absolute magnitude (see below). The greatest uncertainty in the Downes \& Duerbeck (2000) study is the size of the interstellar reddening to individual novae. This uncertainty adds vertical (magnitude) scatter to the data, but it cannot selectively hide faint, fast novae.

The then state-of-the-art photographic studies of the LMC were summarized in Capaccioli et al. (1990). At that time, only 4 novae had well-defined (i.e., directly observed, and not 


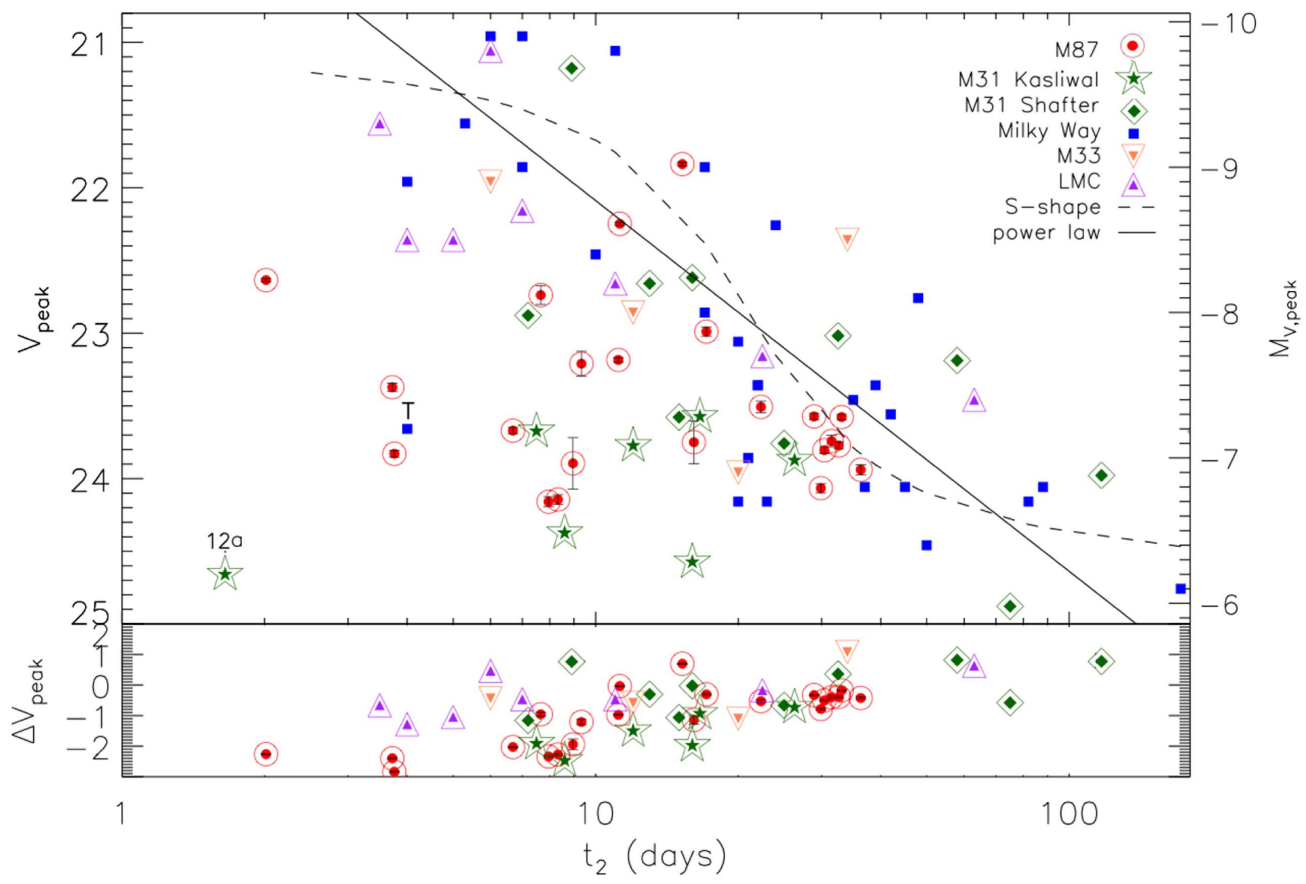

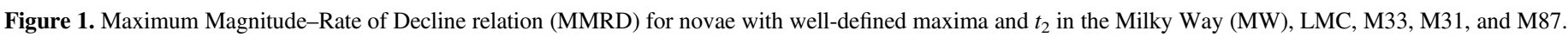

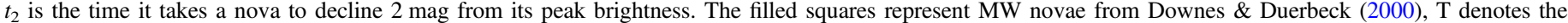

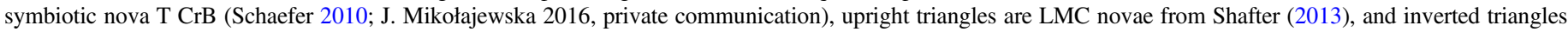

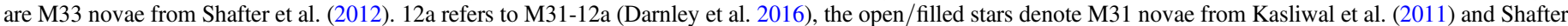

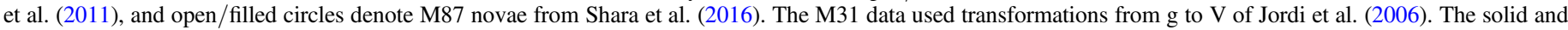

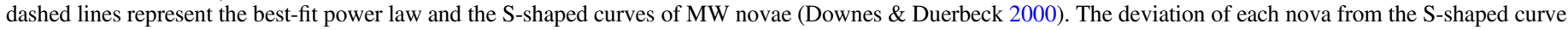
in Figure 1 is plotted in the figure's lower panel.

extrapolated or guessed at) times and magnitudes at maximum light. That entire sample, including the large majority of novae with extrapolated maximum magnitudes and rates of decline, did not detect faint, fast novae. As already noted, Hearnshaw et al. (2004) expressed doubts about the MMRD on the basis of the new observations of fast LMC novae. The most recent summary of LMC novae is that of Shafter (2013). Four more novae with well-defined times of maximum light (within 2 days), maximum magnitudes, and decline times have been observed in the 23 years since Capaccioli et al. (1990). These 8 well-observed LMC novae are included in our figures in the following sections.

The only long baseline, high cadence, CCD-based survey of the Magellanic Clouds is that of Mróz et al. (2016). Of their 15 novae with extremely well-defined decline times, 5 fall in the faint-fast regime (particularly LMCN 2010-11a and LMCN 2012-03a). Unfortunately their CCD saturates in the magnitude range of 11-12, depending on seeing (P. Mroz 2016, private communication). To be conservative we do not include the Mróz et al. (2016) data in our figures.

Capaccioli et al. (1989) summarized the former state-of-theart photographic studies of M31 novae. Unfortunately, the original photometry has not been published, so it is impossible to judge how far they have extrapolated the maximum magnitudes or how well determined are the rates of decline. Faint, fast novae are absent from the data. The Shafter et al. (2011) spectrographic survey of M31 novae summarized the previous decade's photometry of the best studied objects. We include 11 novae with well-defined V-band maxima and $t_{2}$ in our figures. Kasliwal et al. (2011) used the robotic Palomar 60 inch telescope to sample M31 in single (g) filter images in 2008 and 2009, with high cadence and spectroscopically confirmed several of the transients they discovered as classical novae. We adopt the "best-observed" sample of six faint, fast novae of Kasliwal et al. (2011) for comparison with our own HST observations of M87 novae.

We carried out daily Hubble Space Telescope/Advanced Camera for Surveys (HST/ACS) imaging of the giant elliptical galaxy M87 in the F606W (V band) and F814W (I band) filters taken for HST Cycle-14 program 10543 (PI-E. Baltz) over the 72 day interval 2005 December 24 through 2006 March 5, with a few 5 day gaps at the end of the run. Full details of the observations, data reductions, detections, and characterizations of 32 certain and 9 likely novae are given in Shara et al. (2016). Figures 1 and 2 of that paper include the daily images of each nova, and their full light and color curves, respectively. This survey for extragalactic novae is unprecedented, because $H S T$ observations rule out gaps due to weather and there are no variations in limiting magnitude due to variable seeing or lunar phase. Thus, 21 novae were detected both before and after maximum light, and their brightnesses were measured within $12 \mathrm{hr}$ of maximum light. Our daily sampling over a 10 week span was deep enough to be almost impervious to M87's background light, revealing novae to within $10^{\prime \prime}$ of the galaxy's nucleus. In addition, novae were detected over a nearly 6 magnitude range of brightness, so even the faintest and fastest of novae were easily detected.

\section{Milky Way, LMC, M33, M31, and M87 MMRD Data}

In Figure 1 we plot the MMRD diagram of all the Galactic novae with expansion parallax distances from Downes \& Duerbeck (2000). We add T CrB, the symbiotic nova with a similarly reliable distance and absolute magnitude, to the novae. For $\mathrm{T} \mathrm{CrB}, t_{2}$ is taken from Schaefer (2010), while the 


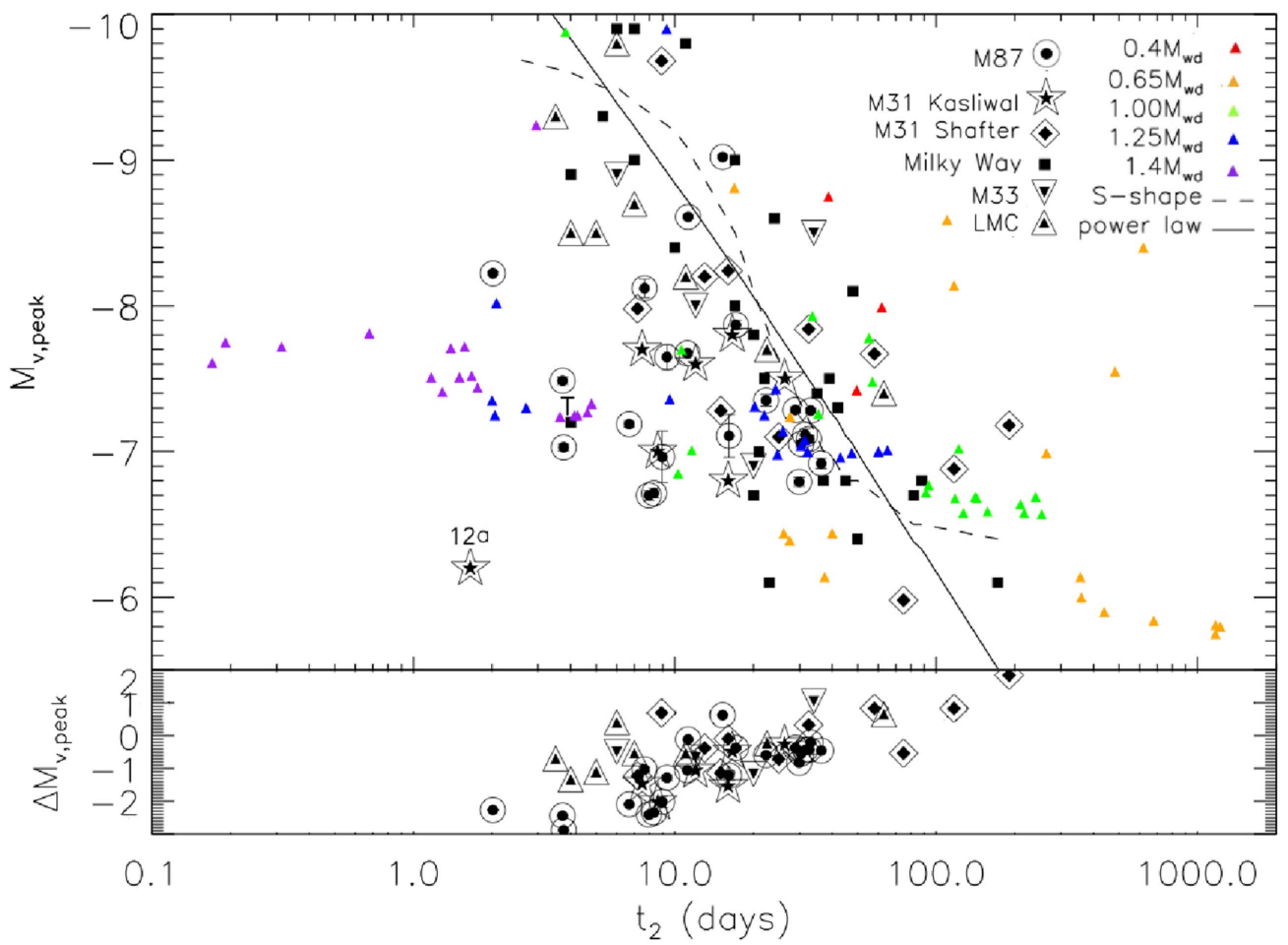

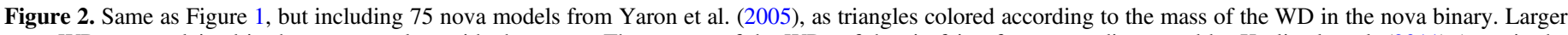

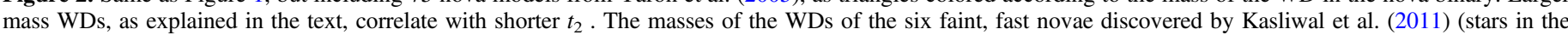

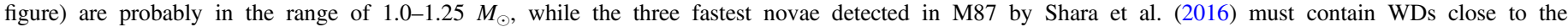
Chandrasekhar mass.

distance is measured from the known radius of its Roche lobefilling red giant, its well-studied orbit, and its angular radius from optical (K-band) interferometry (J. Mikołajewska 2016, private communication). We also plot the most reliable (i.e., with well-determined maximum brightnesses and $t_{2}$ ) novae in the LMC and M33, the rapidly recurring nova M31-12a in M31 (Darnley et al. 2016), the best observed faint-fast novae in M31 (Kasliwal et al. 2011), and the 21 novae from our HST survey of M87.

Our complete sample of M87 novae not only supports the Kasliwal et al. (2011) claim that faint, fast novae exist, but triples the sample of such objects and adds three of the fastest examples known. These three novae, with $t_{2}$ of $2.01,3.72$, and 3.75 days, respectively, are comparable to V597 Pup (Hounsell et al. 2016) and the extraordinary recurrent nova M31-12a in the Andromeda galaxy, which erupts once or twice every year (Henze et al. 2015) and fades by 2 mag in just 1.65 days (Darnley et al. 2016). Few novae in M31 and elsewhere have been seen with similar values of $t_{2}$, but are seen almost always with $M=-9.5$ to -10 rather than with the values of -7 to -8 observed in M87 and in T CrB.

It is clear from Figure 1 that novae, long believed to be "standard candles," display three magnitudes of dispersion in the magnitudes of their MMRD diagrams when high cadence, deep CCD sampling is used so as not to exclude faint, fast novae. They cannot be reliably used to measure extragalactic distances, or the distances of newly discovered Galactic novae. This strengthens the similar conclusion of Ferrarese et al. (2003), albeit based on a smaller and less densely sampled group of nine novae in M49, and by Kasliwal et al. (2011) on the basis of the faint, fast novae they detected in M31.

Why did the roughly 100 Galactic, LMC, SMC, and M31 novae of the previous century and noted in Section 2 provide
MMRDs that yielded a few good extragalactic distances? The surveys that located these objects all suffered from the same incompleteness. The relatively easy-to-find classical novae populate, in zeroth approximation, the upper left and lower right quadrants of MMRD plots. The upper right quadrant is mostly empty (very slow, very luminous novae are rare), while the hard-to-find objects in the lower left quadrant (faint and fast) were all missed. The preferential detection of novae in only the upper left and lower right quadrants suggested a spurious correlation-bright objects are preferentially fast and faint objects are slow. Once the lower left quadrant was filled in (with $40 \%$ of all novae) — as has now happened - the apparent correlation vanished.

\section{Why Is There So Much Scatter in the MMRD Plot?}

We have already noted that the mass of the WD in a nova binary is predicted to be an important parameter in determining how quickly a nova ejects its hydrogen-rich envelope, and thus how fast it declines from maximum light. This is quantifiable via the 75 self-consistent models of novae of Yaron et al. (2005), which not only varied the WD mass, but also varied the WD luminosity and the accretion rate of matter onto WDs. Kasliwal et al. (2011, p. 94) plotted all these models in an $M_{V}$-timescale diagram and concluded that "Some hot and massive WDs with high accretion rates can result in a faint and fast nova population consistent with the P60-FasTING sample." We now show that low accretion rates, and especially low accreted envelopes masses, are equally effective at creating faint, fast, novae on massive WDs.

To clearly separate each of the parameters that determine the location of a nova model in the $M_{V}-t_{2}$ diagram, we superpose onto Figure 1 all 75 of the Yaron et al. (2005) 


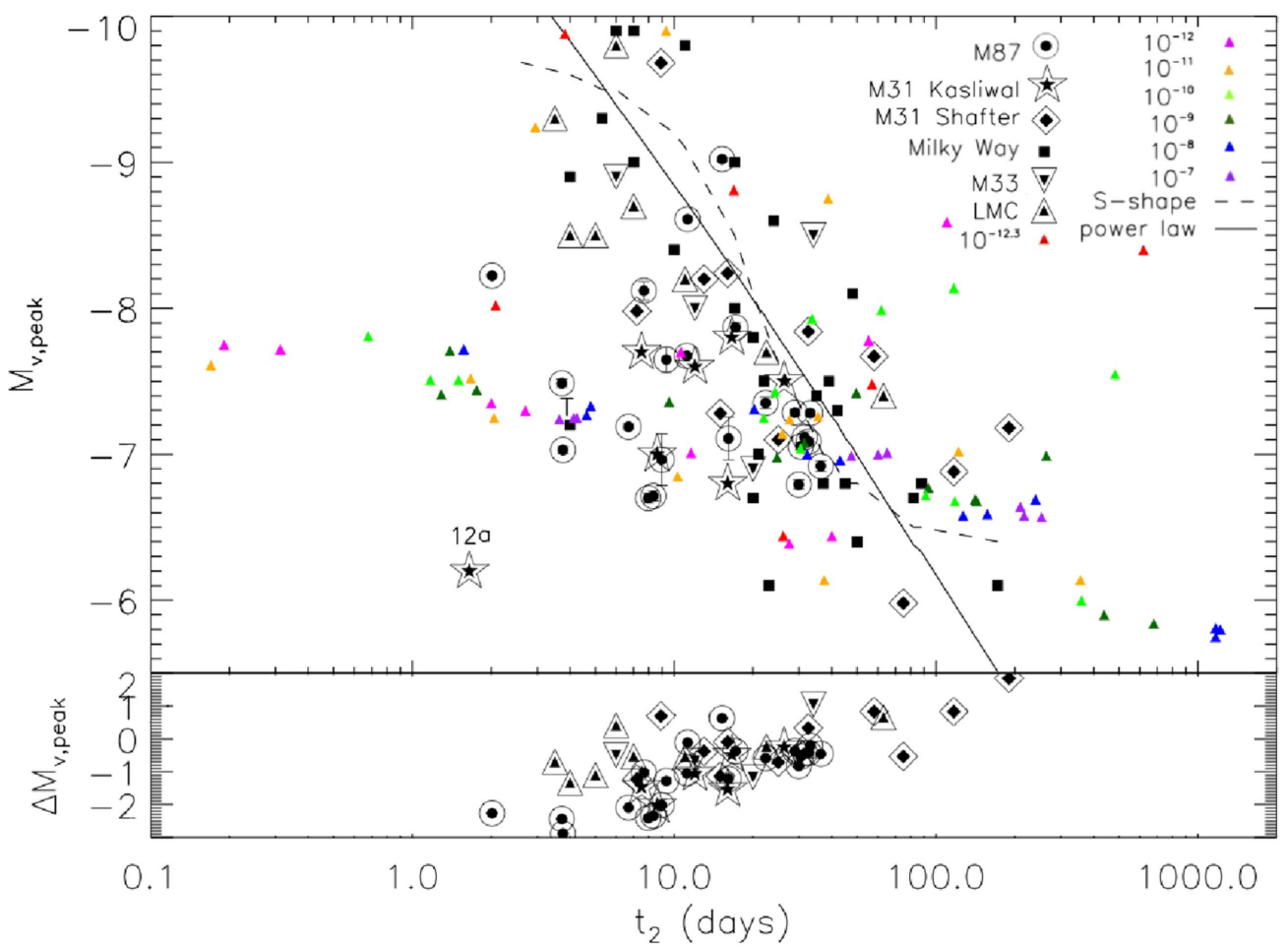

Figure 3. Same as Figure 1, but including 75 nova models from Yaron et al. (2005) represented as triangles colored according to the mass accretion rate (assumed constant) onto the WD. Mass accretion rates that differ by several orders of magnitude can produce identical values of $t_{2}$ or peak luminosity.

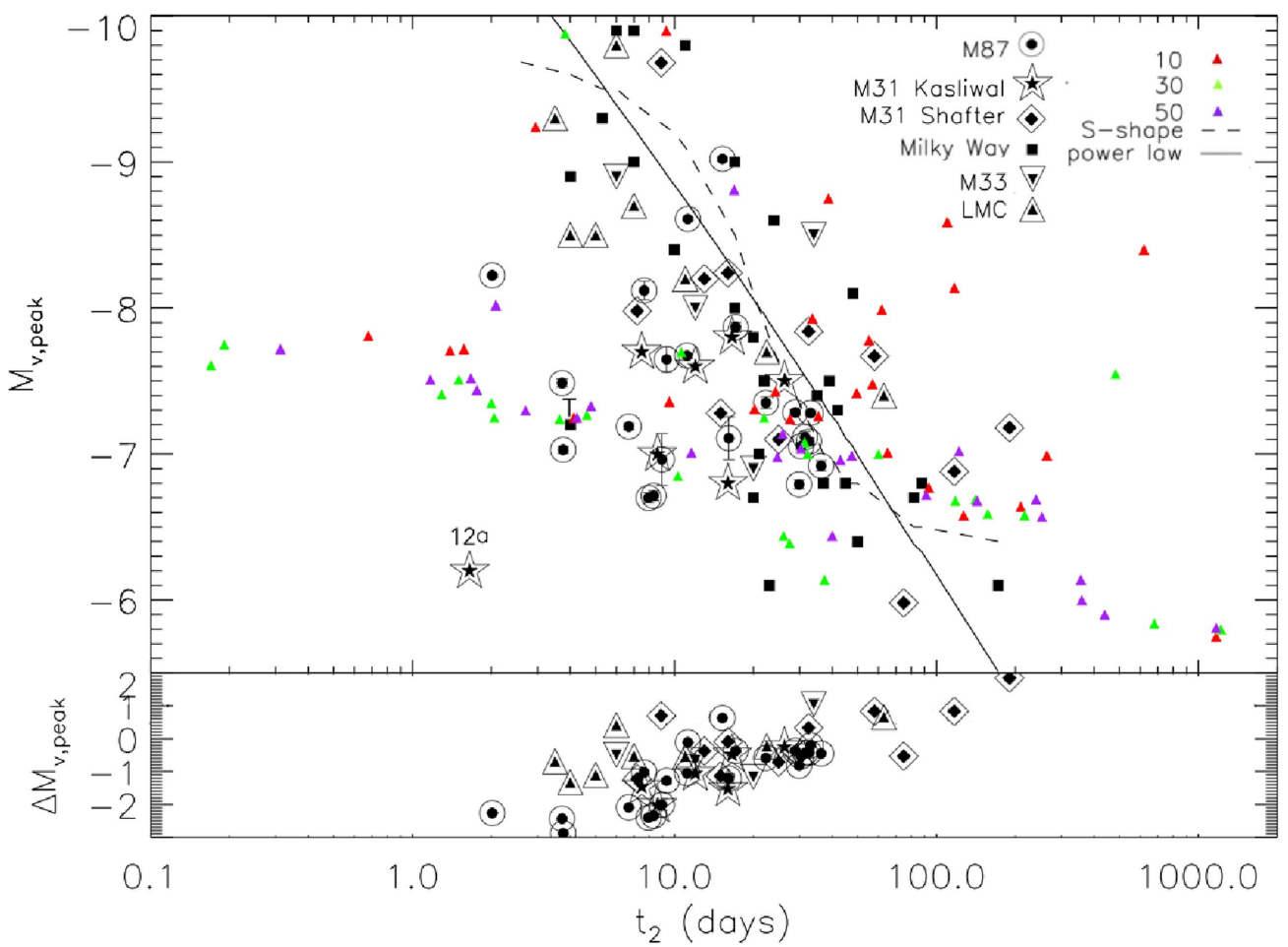

Figure 4. Same as Figure 1, but including 75 nova models from Yaron et al. (2005) represented as triangles colored according to the WD core temperature (in units of millions of Kelvins), and hence the luminosity of the WD in the nova binary.

models, color-coded by WD mass (Figure 2), mass accretion rate (Figure 3), WD core temperature (Figure 4), and total accreted envelope mass (Figure 5). We note that the Yaron et al. (2005) models calculate $t_{3}$ (as the timescale of mass-loss $t_{\mathrm{ml}}$ ) rather than $t_{2}$; we assume that $t_{2}$ is simply two-thirds of $t_{3}$. Like Kasliwal et al. (2011), we assume that novae at maximum luminosity display spectral types close to A5V to convert the Yaron et al. (2005) maximum model luminosities to $M_{V}$. The models depicted in Figure 2 (and those in Hillman et al. 2016) predict that any nova displaying $t_{2}<10$ days must contain a WD with a mass in excess of $1.25 M_{\odot}$. In Kasliwal et al. (2011), 2 of the 6 best-observed M31 novae display $t_{2}<10$ 


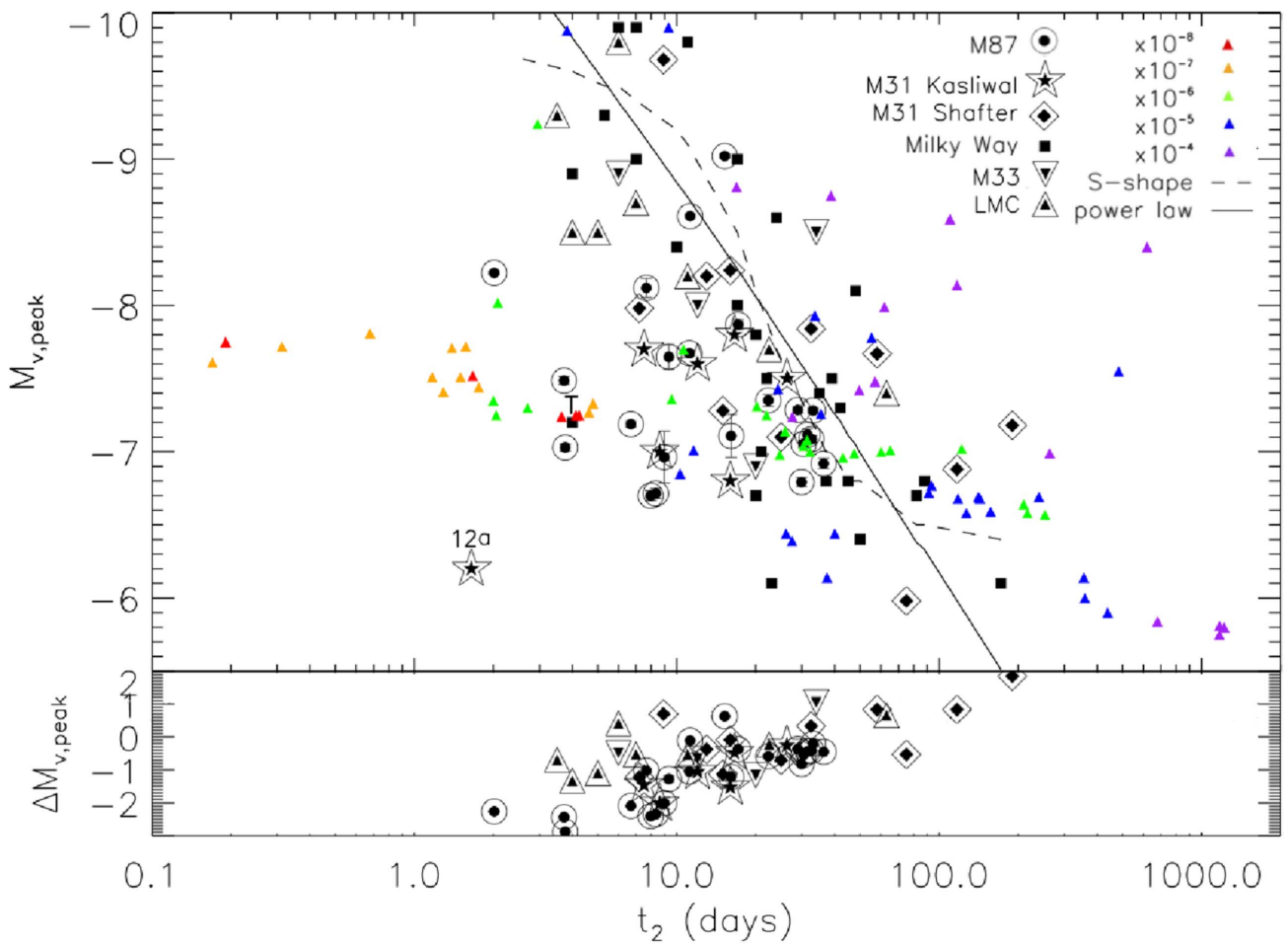

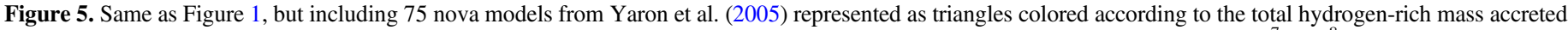

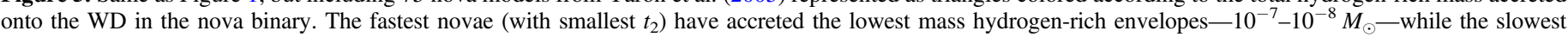

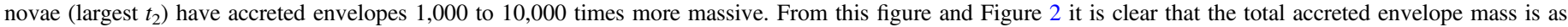
critical as a parameter as the WD mass in determining the peak luminosity and $t_{2}$ of a nova.

days, while 9 of the 21 novae we detected in M87 with welldetermined values of $t_{2}$ display the same results. While the Shara et al. (2016) survey of M87, spanning 72 days, is ineffective at identifying novae with $t_{2}$ longer than 50-60 days, it is clear from their Figure 12 that over $40 \%$ of fast novae display $t_{2}<10$ days. Such objects are certainly not rare, and they reaffirm the claim that WD masses in nova binaries are on average much larger than those in the field (Ritter et al. 1991; Pagnotta \& Schaefer 2014).

It is true that varying the rate of mass accretion onto a WD of a given mass in a nova binary can lead to very different outcomes (Paczynski \& Zytkow 1978; Prialnik et al. 1982). In particular, one might guess that, after the WD mass, the mass accretion rate is the most important parameter determining the properties of a nova. In Figure 3 we again replot the 75 nova models of Yaron et al. (2005) on the observational MMRD diagram of Figure 1, but this time the models are color-coded according to mass accretion rate. In sharp contrast with Figure 2, where it is apparent that WD mass and $t_{2}$ are strongly correlated, Figure 3 demonstrates that the mass accretion rate and $t_{2}$ are not correlated at all. Accretion rates of $10^{-12.3} M_{\odot} \mathrm{yr}^{-1}$ can produce novae with $t_{2}$ as small as 0.2 days or as large as 500 days. Peak luminosities, for this same accretion rate, range from $M_{V}=-6.5$ to -9.8 . Similar large ranges are seen in both $M_{V}$ and $t_{2}$ for other values of the mass accretion rate. A similar result is seen in Figure 4, where we replot the 75 Yaron et al. (2005) models again, but color-coded for WD core temperature (and thus WD luminosity). WD luminosity by itself plays a small role, if any, in determining the luminosities or decline times of novae.

The inconclusive results of Figures 3 and 4 are resolved in Figure 5, where we again plot the Yaron et al. (2005) nova models, but now color-coded according to the mass of the hydrogen-rich envelope accreted before a nova TNR begins. The correlation between $t_{2}$ and accreted envelope mass is evidently much stronger than the correlations of WD luminosity or mass accretion rate with $t_{2}$. This is even more obvious in Figure 6, where we plot the accretion rate, WD temperature, and accreted envelope mass versus $t_{2}$. A useful empirical equation relating these latter two quantities is the least-squares fit straight line

$\log M_{\text {env }}=0.825 \log \left(t_{2}\right)-6.108$.

The underlying reason for the behavior in Figure 6 is simple: the smallest envelope masses can be ejected the most quickly, leading to the smallest observed $t_{2}$.

\section{A Prediction: The Existence and Detection of Ultrafast Novae}

A strong and testable prediction, overlooked until now, emerges from the models of Yaron et al. (2005) that is evident in, and physically understandable from Figures 2, 4, 5, and 6. We predict that WDs with masses close to $1.4 M_{\odot}$, which have slowly $\left(10^{-10}-10^{-12.3} M_{\odot} \mathrm{yr}^{-1}\right)$ accreted envelopes with low mass $\left(10^{-7}-10^{-8} M_{\odot}\right)$ can produce novae with $t_{2}$ as short as $5 \mathrm{hr}$. Simulated light curves of such novae are shown in Figure 4 of Hillman et al. (2014).

We (rather arbitrarily) define an ultrafast nova as one that displays $t_{2}<1$ day. No such nova has ever been observed, but we maintain that this is entirely due to sampling bias. Every ground-based survey for extragalactic novae reported in the past century has employed cadences of a day or longer. Even our own M87 nova survey, which successfully sampled that galaxy daily for 10 weeks without gaps, was unlikely to find any nova that appeared and then faded by 2 mag in less than one day, let alone in 5 hours. 


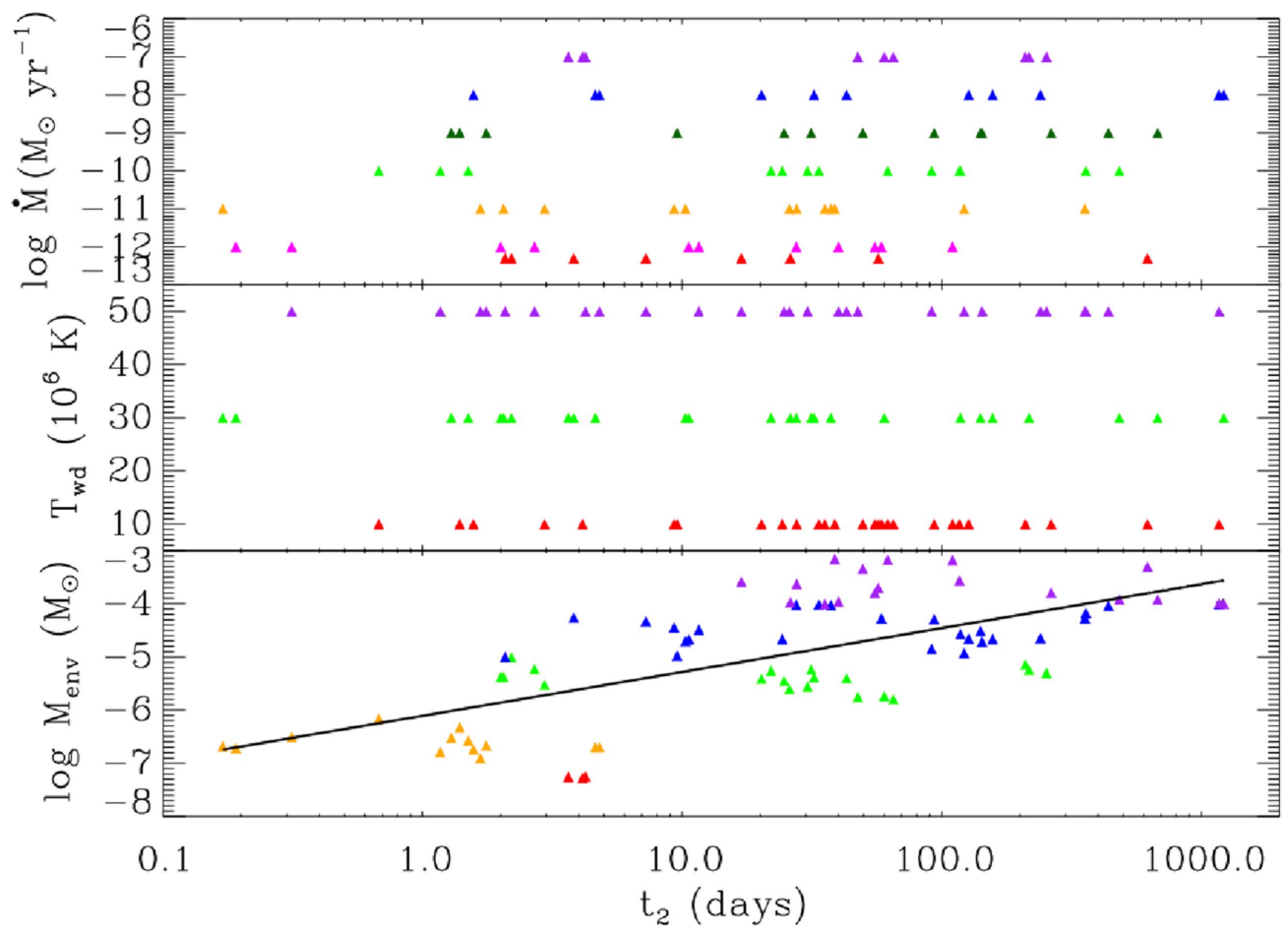

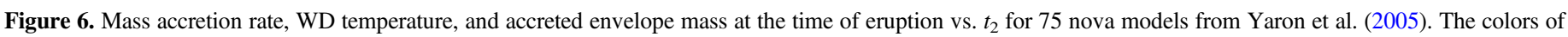

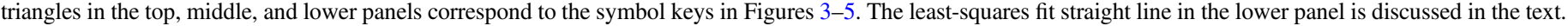

High accretion rates can accumulate critical envelope masses on timescales as short as years (Yaron et al. 2005). This is the source of the RNe, which have massive WDs and inter-eruption intervals of a century or less, e.g., M31-12a and T CrB. RNe have recently been estimated to comprise $25 \%$ of all novae (Pagnotta \& Schaefer 2014). We emphasize that (still hypothetical) ultrafast novae are not RNe. Their low accretion rates must inevitably lead to millennia or longer between their eruptions. If ultrafast novae are eventually detected, we predict that their ejecta will be significantly enhanced in nitrogen relative to the solar value. This is because the long timescale needed to bring these slowly accreted envelopes to the critical mass for initiation of a TNR will allow for significant diffusion of hydrogen into the underlying WD (Kovetz \& Prialnik 1985). This mixing enriches the burning envelope of novae by an order of magnitude or more in $\mathrm{CNO}$ isotopes, which are mostly converted to nitrogen by the TNR.

RNe do not fit the classical nova MMRD (Schaefer 2010). But if astronomers are to use MMRD, and to have any confidence in the use of the MMRD for novae discovered in the future, one must be able to distinguish a newly discovered nova as being an $\mathrm{RN}$ or a classical nova. (By $\mathrm{RN}$ we adopt the conventional definition: an $\mathrm{RN}$ erupts at least twice per century.)./ Pagnotta \& Schaefer (2014) have exhaustively researched this topic and have demonstrated that the only certain diagnostic of a nova being a $\mathrm{RN}$ is observing a second outburst. Thus any newly discovered, fast Galactic, or extragalactic nova could be faint and fast (and relatively close) or luminous and fast (and relatively distant). MMRD alone cannot yield a reliable distance for any nova with $t_{2}<30$ days. Slower novae all display $M=-6.5 \pm 0.5 \mathrm{mag}$, but this is almost independent of $t_{2}$ and the MMRD.

Are ultrafast novae rare? A reliable theoretical prediction of the frequency of ultrafast novae relative to all other novae in a galaxy would involve a population synthesis model that produces novae from an initial and evolving binary population and self-consistently calculates the time-dependent mass transfer rate to the WD in each nova system over that system's lifetime. This is a challenging problem, far beyond the scope of this paper. A much simpler approach is to observationally detect ultrafast novae and measure their relative frequency among all novae.

How might ultrafast novae be detected? The answer is straightforward: via surveys of nearby galaxies with cadences of the order of one hour, rather than days. Figure 2 demonstrates that ultrafast novae should achieve $M_{V}$ of -6.5 to -7.5 , corresponding to 17-18th magnitude in M31. Detecting such rapid transients, and following them down to 20th magnitude is within the reach of modern CCD cameras attached to $0.5 \mathrm{~m}$ aperture telescopes. The Zwicky Transient Facility will utilize a large format camera and the Samuel Oschin 48 inch Palomar Schmidt telescope to begin imaging about 3750 square degrees an hour to a depth of 20.5-21 mag in 2017. With a $1 \mathrm{hr}$ cadence it should easily discover ultrafast novae in Local Group galaxies. Confirmation, via spectroscopy or narrowband-broadband imaging, can be done in the days following the detection of rapid transient candidates, as novae remain bright in $\mathrm{H} \alpha$ for much longer than they do in continuum light (Ciardullo et al. 1983; Neill \& Shara 2004).

\section{Summary and Conclusions}

Of the 21 well-observed novae in M87, 9 display $t_{2}$ brightness decline times under 10 days, and 3 more have $t_{2}<4$ days. These novae are up to 3 mag fainter than what was predicted by the MMRD relation, and are similar to the "faint, fast novae" first detected by Kasliwal et al. (2011) in M31. The fact that these novae are both common and ubiquitous demonstrates that complete samples of extragalactic novae are not reliable standard candles, and that the MMRD should 
not be used in the era of precision cosmology either for cosmic distance determinations or the distances of Galactic novae.

The Yaron et al. (2005) models of novae explain faint, fast novae as those that occur on very massive WDs with very lowmass envelopes. Low-mass envelopes that were accreted quickly lead to RNe. We predict that those accreted slowly yield (previously overlooked) ultrafast novae that brighten and fade by 2 mag in under 1 day. Such ultrafast novae are also predicted to display large nitrogen enhancements relative to the solar value. We predict that surveys of M31 and other nearby galaxies with cadences of about $1 \mathrm{hr}$ will reveal these novae, even with modest-sized telescopes.

We gratefully acknowledge the support of the STScI team responsible for ensuring timely and accurate implementation of our M87 program. Support for program \#10543 was provided by NASA through a grant from the Space Telescope Science Institute, which is operated by the Association of Universities for Research in Astronomy, Inc., under NASA contract NAS 5-26555. This research has been partly supported by the Polish NCN grant DEC-2013/10/M/ST9/00086. M.M.S. gratefully acknowledges the support of Ethel Lipsitz and the late Hilary Lipsitz, longtime friends of the AMNH Astrophysics department.

\section{References}

Arp, H. C. 1956, AJ, 61, 15

Baade, W. 1944, ApJ, 100, 137

Bird, S., Harris, W. E., Blakeslee, J. P., \& Flynn, C. 2010, A\&A, 524, A71

Capaccioli, M., della Valle, M., D’Onofrio, M., \& Rosino, L. 1990, ApJ, 360,63

Capaccioli, M., della Valle, M., Rosino, L., \& D’Onofrio, M. 1989, AJ, 97, 1622

Chandrasekhar, S. 1931, ApJ, 74, 81

Chandrasekhar, S. 1935, MNRAS, 95, 207

Christy, R. F. 1966, ApJ, 144, 108

Ciardullo, R., Ford, H., \& Jacoby, G. 1983, ApJ, 272, 92

Ciardullo, R., Jacoby, G. H., Ford, H. C., \& Neill, J. D. 1989, ApJ, 339, 53

Cohen, J. G. 1985, ApJ, 292, 90

Darnley, M. J., Henze, M., Bode, M. F., et al. 2016, ApJ, 833, 149

de Grijs, R., \& Bono, G. 2014, AJ, 148, 17 della Valle, M., \& Livio, M. 1995, ApJ, 452, 704

Downes, R. A., \& Duerbeck, H. W. 2000, AJ, 120, 2007

Faulkner, J., Flannery, B. P., \& Warner, B. 1972, ApJL, 175, L79

Ferrarese, L., Côté, P., \& Jordán, A. 2003, ApJ, 599, 1302

Hachisu, I., \& Kato, M. 2010, ApJ, 709, 680

Hearnshaw, J. B., Livingston, C. M., Gilmore, A. C., \& Kilmartin, P. M. 2004

in ASP Conf. Ser. 310, Variable Stars in the Local Group, ed. D. W. Kurtz \& K. R. Pollard (San Francisco, CA: ASP), 103

Henze, M., Darnley, M. J., Kabashima, F., et al. 2015, A\&A, 582, L8

Hillman, Y., Prialnik, D., Kovetz, A., \& Shara, M. M. 2016, ApJ, 819, 168

Hillman, Y., Prialnik, D., Kovetz, A., Shara, M. M., \& Neill, J. D. 2014, MNRAS, 437, 1962

Hounsell, R., Darnley, M. J., Bode, M. F., et al. 2016, ApJ, 820, 104

Hubble, E. 1929a, ApJ, 69, 103

Hubble, E. 1929b, PNAS, 15, 168

Inno, L., Bono, G., Matsunaga, N., et al. 2016, ApJ, 832, 176

Jordi, K., Grebel, E. K., \& Ammon, K. 2006, A\&A, 460, 339

Kasliwal, M. M., Cenko, S. B., Kulkarni, S. R., et al. 2011, ApJ, 735, 94

Kovetz, A., \& Prialnik, D. 1985, ApJ, 291, 812

Livio, M. 1992, ApJ, 393, 516

Mclaughlin, D. B. 1945, PASP, 57, 69

Mróz, P., Udalski, A., Poleski, R., et al. 2016, ApJS, 222, 9

Neill, J. D., \& Shara, M. M. 2004, AJ, 127, 816

Paczynski, B., \& Zytkow, A. N. 1978, ApJ, 222, 604

Pagnotta, A., \& Schaefer, B. E. 2014, ApJ, 788, 164

Prialnik, D., \& Kovetz, A. 1984, ApJ, 281, 367

Prialnik, D., \& Kovetz, A. 1995, ApJ, 445, 789

Prialnik, D., Livio, M., Shaviv, G., \& Kovetz, A. 1982, ApJ, 257, 312

Riess, A. G., Macri, L., Casertano, S., et al. 2011, ApJ, 730, 119

Ritter, H., Politano, M., Livio, M., \& Webbink, R. F. 1991, ApJ, 376, 177

Sandage, A. R. 1971, in Study Week on Nuclei of Galaxies, ed.

D. J. K. O'Connell (Amsterdam: North Holland), 601

Schaefer, B. E. 2010, ApJS, 187, 275

Shafter, A. W. 2013, AJ, 145, 117

Shafter, A. W., Darnley, M. J., Bode, M. F., \& Ciardullo, R. 2012, ApJ, 752,156

Shafter, A. W., Darnley, M. J., Hornoch, K., et al. 2011, ApJ, 734, 12

Shara, M. M. 1981, ApJ, 243, 926

Shara, M. M., Doyle, T. F., Lauer, T. R., et al. 2016, ApJS, 227, 1

Shara, M. M., Prialnik, D., \& Shaviv, G. 1980, ApJ, 239, 586

Starrfield, S., Iliadis, C., \& Hix, W. R. 2016, PASP, 128, 051001

Starrfield, S., Sparks, W. M., \& Truran, J. W. 1986, ApJL, 303, L5

Starrfield, S. G., Sparks, W. M., \& Truran, J. W. 1975, MSRSL, 8, 425

van den Bergh, S. 1992, PASP, 104, 861

Warner, B. 1995, Cataclysmic Variable Stars (Cambridge: Cambridge Univ. Press)

Yaron, O., Prialnik, D., Shara, M. M., \& Kovetz, A. 2005, ApJ, 623, 398

Zwicky, F. 1936, PASP, 48, 191 